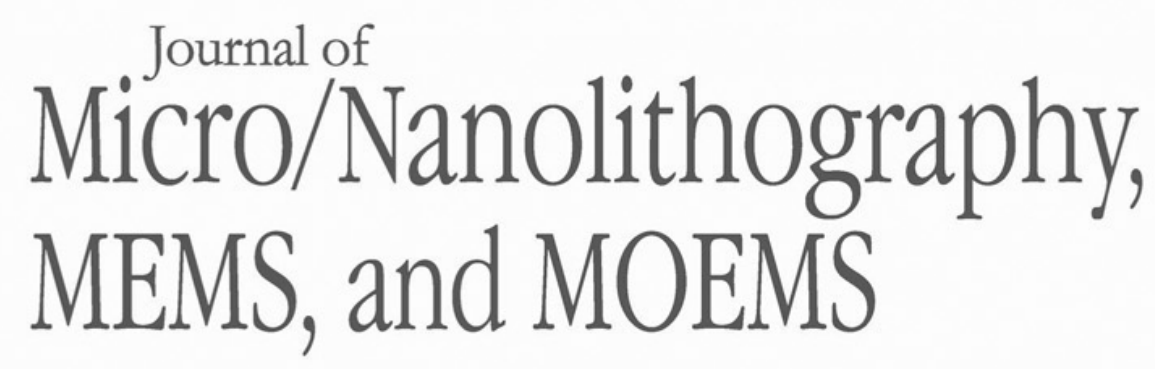

Nanolithography.SPIEDigitalLibrary.org

\title{
2019 List of Reviewers
}


The Journal of Micro/Nanolithography, MEMS, and MOEMS would like to sincerely thank the following individuals who served as reviewers in 2019. The success of our publication hinges on the voluntary contributions of time and energy put forth by these professionals.

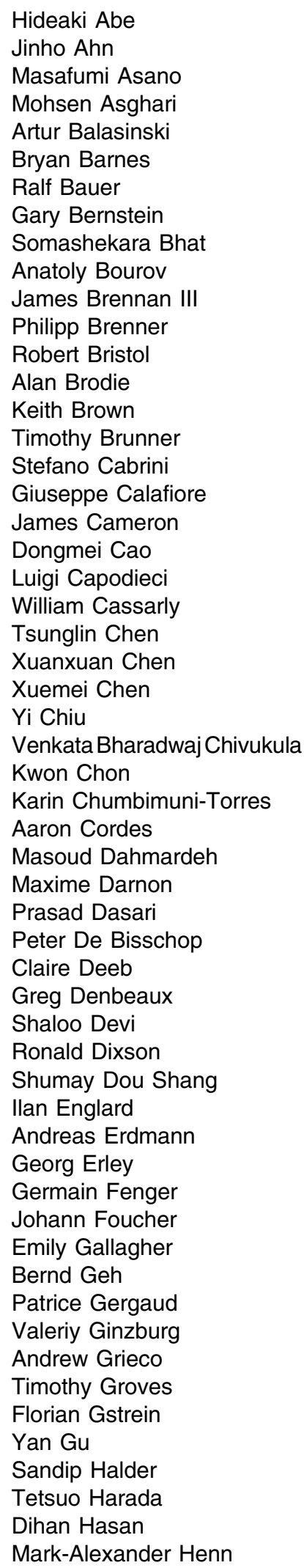

Hideaki Abe

Masafumi Asano

Mohsen Asghar

Balasinski

Ralf Bauer

Gary Bernstein

a Bhat

James Brennan III

Keith Brown

Timothy Brunne

James Cameron

Dongmei Cao

Luigi Capodieci

Xuemei Chen

Kwon Chon

Masoud Dahmardeh

Maxime Darnon

Claire Deeb

Greg Denbeaux

Shumay Dou Shang

Ilan Englard

Georg Erley

Germain Fenge

Johann Foucher

Emily Gallaghe

Patrice Gergaud

Valeriy Ginzburg

Yan Gu

Sandip Halder

Dihan Hasan

Mark-Alexander Henn
Craig Higgins

Shannon Hill

Hartmut Hillmer

Ulrich Hofmann

Jingtian $\mathrm{Hu}$

Thomas Huisman

Stefan Hunsche

Soichi Inoue

Abid lqbal

Ningning Jia

Pinggang Jia

Fan Jiang

Sumit Jindal

Taher Kagalwala

Chih-Ming Ke

Kahraman Keskinbora

Ryoung-han Kim

SeongSue Kim

Marie Krysak

Ashish Kumar

Varun Kumar

Neal Lafferty

Michele Laus

Chang-Chun Lee

Soo-Young Lee

Harry Levinson

Fengyuan Li

Lijie Li

Peng Li

Xiaohai Li

Ted Liang

Yichen Liang

Chichun Liu

Huicong Liu

Ke Liu

Rui Liu

Jordi Llobet

Daniel Lopez

Gian Lorusso

Eric Louis

Rajesh Luharuka

Larry Luo

Xu Ma

Ruben Maas

Chris Mack

Chiatto Matteo

Moyra McManus

Henry Megens

Lawrence Melvin, III

Sascha Migura

Marshal Miller

Paul Mirkarimi

Chad Mirkin

Hiroyuki Miyazoe

Boris Mizaikoff

Yasutaka Morikawa
Nezih Mrad

Sameer Mulani

Jan Mulkens

Viviana Mulloni

Mark Neisser

Christopher Ober

Ndubuisi Orji

Prem Pal

Piyush Pathak

John Petersen

Michael Postek

Lingling $\mathrm{Pu}$

Zhenyun Qian

Lei Qiao

Manasi Raje

Ivo Rangelow

Alan Rosenbluth

Alon Rosenthal

Ildar Salakhutdinov

Amin Sandoughsaz

Iqbal Saraf

Marc Schnieper

Frank Scholze

Bernd Schulz

Kazunori Seki

Apo Sezginer

Akshdeep Sharma

Abraham Slachter

Mark Smith

LaVern Starman

Bo Su

Vishnu-Baba Sundaresan

Makoto Suzuki

N. Kumar Swamy

Zishan Ali Syed Mohammed

Tanguy Terlier

Brad Thiel

Chuan-Kang Ting

Juan Torres

Illhami Torunoglu

Istvan Ulbert

Nilesh Vasa

John Villarrubia

Andras Vladar

Marko Vogler

Justin Wan

Dapeng Wang

Fei Wang

Hao Wang

Peng Wang

Wentao Wang

Takeo Watanabe

Rich Wise

Qiang Wu

Jiwen Xiang

Hong Xiao 
2019 List of Reviewers

Jin Xie

Kaikai Xu

Kenji Yamazoe
Bin Yang

Longfei Yang

Xiaodong Yang
Yansheng Zhang

Jinlong Zhu 